\title{
Exercise program implementation proves not feasible during acute care hospitalization
}

\author{
Cynthia J. Brown, MD, MSPH; ${ }^{1-2^{*}}$ Claire Peel, PhD, PT; ${ }^{3}$ Marcas M. Bamman, PhD; ${ }^{4}$ Richard M. Allman, MD $^{1-2}$ \\ ${ }^{1}$ Birmingham/Atlanta Department of Veterans Affairs Geriatric Research, Education, and Clinical Center, Birming- \\ ham, AL; ${ }^{2}$ Department of Medicine, ${ }^{3}$ School of Health Professions, and ${ }^{4}$ Department of Physiology and Biophysics, \\ University of Alabama at Birmingham, Birmingham, $A L$
}

\begin{abstract}
Functional decline during hospitalization occurs in up to $65 \%$ of older adults. This study determined the feasibility of an inpatient followed by an in-home exercise program for patients with limited ambulatory ability on hospital admission. Patients aged $\geq 60$ years who were admitted to the hospital with an acute medical illness associated with limited ambulatory ability were eligible for the study. Of 76 eligible patients, 10 were recruited, with only 1 patient completing the 24-week exercise program. Barriers to recruitment included illness severity, short hospital stays, and patient refusal. Hospital readmission during the in-home exercise program occurred for three of the seven exercise group participants. In the exercise group, four of the seven patients participated in at least 3 weeks of exercise posthospitalization. Qualitative interviews suggested most patients believed exercise to be beneficial, but this interest did not translate into adherence to this study protocol. Initiation of an inpatient exercise program was not feasible in the study population. The in-home program was more feasible but target criteria need refinement.
\end{abstract}

Key words: activities of daily living, exercise, feasibility studies, frail elderly, geriatrics, hospitalization, recovery of function, rehabilitation, veterans, walking.

\section{INTRODUCTION}

A decline in physical function during hospitalization for acute illness is common in older adults [1-7]. In a study by Lamont et al., 75 percent of the 60 functionally independent persons over 75 years of age admitted to the hospital for an acute illness were no longer independent at discharge, and 15 percent were discharged to nursing homes [1]. In a group of persons 74 years or older admitted to the medicine service of a university-affiliated hospital, 65 percent of patients experienced a decline in their activities of daily living (ADL) (transferring, toileting, feeding, mobility, and grooming) by the second day of hospitalization, with no improvement by discharge [2]. An additional 10 percent had declined by discharge [2]. In a report of 500 older male veterans hospitalized for an acute medical illness, a significant decline in physical performance was noted from admission to discharge for the entire study population [3]. At 1-year postdischarge, physical function had improved but had not returned to preadmission levels for 54 percent of the study participants

Abbreviations: $\mathrm{ADL}=$ activities of daily living, APS $=$ Acute Physiology Score, BVAMC = Birmingham Department of Veterans Affairs Medical Center, CPRS = computerized patient record system, IRB = institutional review board, PI = principal investigator, $\mathrm{RCT}=$ randomized controlled trial, $\mathrm{SD}=$ standard deviation, VA = Department of Veterans Affairs.

*Address all correspondence Cynthia J. Brown, MD, MSPH; VAMC GRECC, 11-G, Room 8226, 1530 3rd Avenue South, Birmingham, AL 35294-0001; 205-934-9261; fax: 205-558-4749. Email: cbrownmd@uab.edu

DOI: 10.1682/JRRD.2006.04.0034 
[3]. These reports demonstrate that a high probability exists that older adults hospitalized for acute medical conditions will experience a decline in physical function with many failing to recover even during the posthospitalization period.

In response to this problem, we designed and conducted a pilot project to study the feasibility of an exercise program for older adults with limited ambulatory ability when admitted to the hospital. The original pilot study was a randomized controlled trial (RCT) involving an exercise group and a control group, with a planned enrollment of 50 subjects. The exercise group was to receive an intensive exercise program consisting of progressive ambulation, transfer training, and resistance training during hospitalization and during the 6 months posthospitalization. The control group was to receive routine medical care. In addition to assessments on enrollment in the study, follow-up assessments were planned for hospital discharge, and at 6, 12, and 24 weeks postdischarge.

Our primary measure of feasibility was the ability to identify, recruit, and enroll subjects. Secondary measures of feasibility included the ability of subjects to complete two 45-minute sessions of exercise each day during the inpatient phase and subjects' adherence to the in-home exercise program.

This article describes the feasibility of an exercise program for older adults recovering from acute medical conditions, including the need for multiple revisions in the original inclusion criteria and identification of barriers to subject enrollment. We also describe the results of a follow-up study using qualitative methods conducted to further explore barriers to exercise in the management of acute medical conditions. This information will be useful for researchers and clinicians who provide exercise to older adults both during and after hospitalization.

\section{METHODS}

\section{Feasibility of Randomized Controlled Trial}

Described below is the study that we attempted. Our initial goal was to examine the feasibility of a RCT of exercise intervention in patients with acute medical illness. If successful, we had planned to expand this into a large intervention trial. Informed consent and all study protocols were approved by the institutional review board (IRB) of the Birmingham Department of Veterans Affairs Medical Center (BVAMC).

\section{Criteria for Subject Inclusion}

Subjects were $\geq 60$ years old and admitted to the BVAMC for treatment of an acute medical condition. Subjects were to be reimbursed for the time they devoted to participation in the study. The final criteria for inclusion were-

1. Self-reported need for assistance from another person to ambulate or ambulation limited to less than $150 \mathrm{ft}$ at a walking velocity less than $30 \mathrm{~m} / \mathrm{min}$ (unable to walk fast enough to safely cross a street). This criteria is based on the Functional Independence Measure and would equal a score of $\leq 6$.

2. Self-reported history of independent ambulation within 2 weeks of hospitalization.

3. Life expectancy $>1$ year.

4. Medical status that permitted participation in an exercise program.

5. Score of $\geq 17$ on the Mini-Mental State Examination [8] if educational level was grade 8 or less, or score of $\geq 24$ if educational level was greater than grade 8 .

6. Hospitalization expected to continue for at least 5 days after enrollment.

7. Willing and able caregiver at home.

8. Residence within 75 miles of the BVAMC.

\section{Criteria for Subject Exclusion}

Subjects were excluded if their primary reason for hospital admission was a condition for which a standardized exercise program exists, such as myocardial infarction or stroke. Subjects were also excluded for presence of any of the following: unstable angina, a contagious infection, or any medical condition for which ambulation was contraindicated.

\section{Procedures for Subject Recruitment}

Subjects were recruited from patients admitted to the Medicine Service of the BVAMC. The following process was used to identify, recruit, and enroll subjects: using the computerized patient record system (CPRS), we excluded patients who were $<60$ years of age, as well as patients who lived more than 75 miles from the BVAMC, had a diagnosis of dementia, or had a medical condition that would preclude participation in the study (including comatose condition, residence in a nursing home, or being bed or wheelchair bound). 
For all other patients, the project coordinator consulted the nursing staff to solicit information about the patient's current medical status, such as acute confusion, other changes in health status not yet reflected in the medical records, and discharge plans. Nurses were chosen as they are more likely to have up-to-date clinical information about potential participants, which allowed us to assess the patient's eligibility prior to interviewing the patient. Potential participants were visited and verbal assent was obtained for participation in the study. The patient's cognitive status and self-reported ambulation status, both current and past, were assessed to determine if inclusion criteria were met. After meeting all inclusion criteria, written informed consent was obtained from the participant and verbal approval obtained from the physician.

\section{Testing Procedures}

A series of tests was administered to all subjects at enrollment into the study. These tests included the Timed Up and Go [9], strength testing using a handheld dynamometer, the Life-Space Assessment [10], and the 15item Geriatric Depression Scale [11]. The Timed Up and Go involves rising from an armchair, walking $3 \mathrm{~m}$, turning, walking back, and sitting down in the chair [9]. Participants are timed as they perform this activity, and based on multiple studies, a score of $<10 \mathrm{~s}$ is considered normal for older persons and indicates no mobility impairment [12]. The Life-Space Assessment measures mobility within the home and community. Life-space is defined as the area in which one travels over a 4-week period and ranges from being confined to one's bedroom to travel outside of one's community. A score of 60/120 is considered average for older persons [10].

\section{Chart Review Data Collected}

Using CPRS, we collected demographic data including age, sex, race, number of discharge medications, and data to determine the Acute Physiology Score (APS) of the Acute Physiology and Chronic Health Evaluation II [13] and the Charlson Comorbidity Index [14] for use as global measures of illness severity and comorbidity, respectively. The APS uses weighted physiological derangements to determine severity of illness. The total number of points gives a score ranging from 0 to 48, with an increasing score representing a greater severity of illness. The Charlson characterizes the extent of comorbidity by weighting the disease for its severity with higher scores indicating more serious comorbidity.

\section{Proposed Program for Exercise and Control Groups}

The original plan included inpatient exercise sessions twice a day, 7 days a week. Sessions were to include progressive ambulation, transfer training, and strength training. After discharge, subjects were asked to walk 20 to 30 min each day and to perform resistance exercise every other day. Patients were to perform the same exercises for the inpatient and outpatient resistance exercise programs. The prescribed program included five exercises: squat, heel raise, ankle dorsiflexion, overhead press, and elbow flexion. Resistance was provided by a weighted vest for the squat and heel raise and by rubber tubing designed to provide varied levels of resistance for the other three exercises. Patients received training logs that described the exercises to be performed, the amount of resistance, and number of repetitions to be done for each of the five exercises. To facilitate the transition to the home, an exercise trainer made home visits once a week during the first month after discharge, every 2 weeks for the next 2 months, and then once a month for the final 3 months. The trainer, who had extensive experience implementing and evaluating exercise programs for older, communitydwelling adults, facilitated the exercise session, answered questions about the program, and reported any problems to the principal investigator (PI).

Routine medical care was provided to subjects in the control group, which included physical therapy if a consult was initiated by the patient's physician. If referred to physical therapy, patients typically are seen once a day with coverage limited to weekdays. Due to short lengths of stay, the therapist usually focuses on recommendations for discharge destination and equipment needed for ambulation, rather than providing intensive exercise treatment. To control for the attention that the members of the exercise group received, we planned for subjects in the control group to receive daily "friendly visits" by members of the research team. After discharge from the hospital, the control group was to receive phone calls at the same times that the exercise group was visited by the home trainer.

\section{Feasibility of Subject Recruitment and Exercise Adherence}

We documented the number of patients who met inclusion criteria and the number of subjects who were enrolled from March 2003 to April 2004. We recorded reasons for subjects not meeting inclusion criteria and 
changes that were made to the inclusion criteria over the course of the study.

We used the number of sessions of inpatient exercise completed per day to determine the feasibility of an inpatient exercise program.

We used home exercise logs with each of the five exercises performed, amount of resistance used, time spent walking, and number of exercise sessions performed each week to determine the patient's adherence with the home exercise program. These logs were collected and returned by the exercise trainer or mailed by the participant to the PI.

\section{Description of Qualitative Follow-Up Study}

Given the difficulty we experienced recruiting participants and conducting the study activities as originally planned, we conducted a qualitative follow-up study to determine why it was challenging to engage older adults in an exercise program during and after hospitalization. After receiving IRB approval, two groups were sent letters asking permission for a brief telephone interview to discuss their thoughts about the exercise program. Participants of the original study, both exercise and control patients, were invited to participate. In addition, a group of veterans who had been eligible but declined were matched to those who participated, based on date of admission. We used a semistructured interview guide to ask about reasons for being interested or not interested in the exercise program, perceived ability to perform the exercise program, and thoughts about what types of exercise would appeal to other veterans during and after hospitalization.

\section{RESULTS}

Over a 13-month period, we successfully recruited (obtained signed consent forms from) only 10 subjects. Throughout the study period, we made several IRBapproved protocol changes to address barriers to recruitment. Table 1 presents the barriers we noted and the protocol changes made in an effort to address these barriers. The Figure provides a summary of subject recruitment and enrollment. A significant number of patients (21\%) were disqualified because of a medical condition. Table 2 presents data regarding the presence of medical conditions, which resulted in exclusion from the study. Of those disqualified for medical conditions, 40 percent had documented dementia or confusion.

A total of 76 patients met the inclusion criteria, with only 10 consenting to participate in the study. The major reason given for declining participation in the study was "not feeling like exercising" or "not believing that they could exercise."

Of the 10 patients who were successfully recruited, 1 patient withdrew prior to baseline testing. Of the remaining 9 patients, 7 were randomly assigned to the exercise group and 2 to the control group. Table 3 presents the

Table 1.

$\underline{\text { Protocol changes made to address barriers to study recruitment. }}$

Barrier

Patients agreed to participate but discharged prior to informed consent.

Patients discharged prior to completion of baseline testing.

Participants unable to return to the BVAMC for testing due to transportation difficulties.

Unable to recruit despite multiple changes to protocol.
Protocol Change

Changed maximum distance to participants' homes from hospital. Originally maximum distance was 30 miles, then 50 miles, and ultimately 75 miles from Birmingham Department of Veterans Affairs Medical Center (BVAMC).

Allowed informed consent to be signed after discharge if patient indicated verbal interest during admission and agreed to schedule in-home visit to obtain informed consent.

Protocol changed to allow baseline testing to be done in patient's home after discharge.

Member of research team performed follow-up testing in participant's home.

Qualitative phone interview to ask those who were eligible for the study why they did or did not participate and what type of exercise might interest them after hospitalization. 
baseline characteristics and functional status measures for the nine patients. The mean age \pm standard deviation (SD) of the cohort was $70.2 \pm 3.1$, and 78 percent were white. The group was not severely ill on admission, with a mean \pm SD APS [13] of $3.4 \pm 1.9$, but had a moderate number of comorbidities, with a mean Charlson score [14] of $4.3 \pm$ 2.3. The group had significant ambulatory speed impairments as demonstrated by a mean Timed Up and Go [9] score of $18.9 \pm 6.1$, with a range from 8.0 to 26.0. A mean

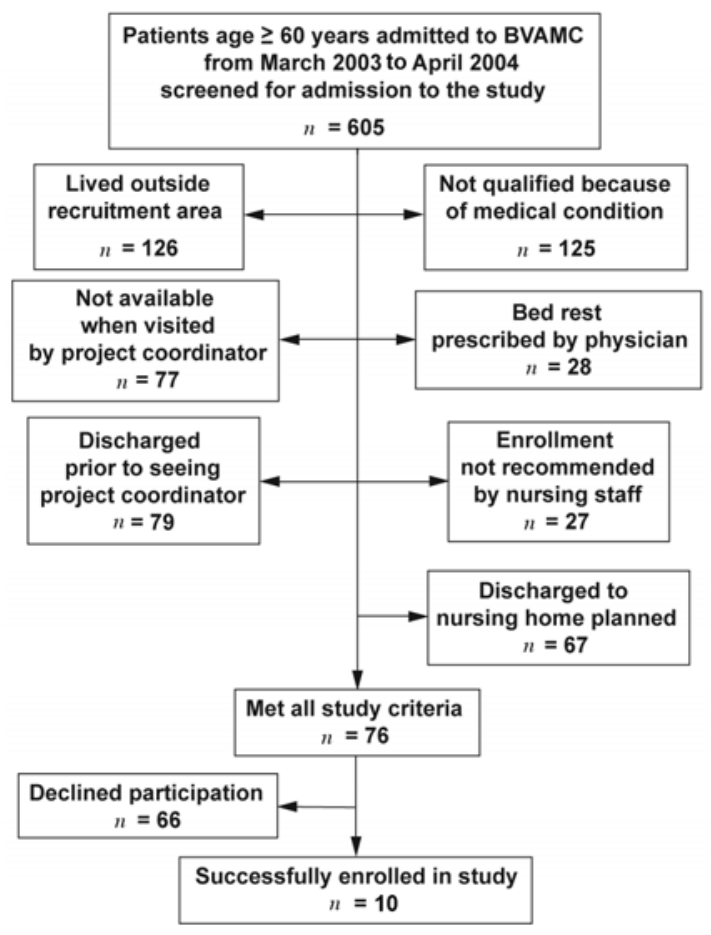

Figure.

Flow of potential participants. Figure shows number of patients, age $\geq 60$ years, admitted to Birmingham Department of Veterans Affairs Medical Center (BVAMC) who were screened for exercise study and either enrolled, declined, or were not eligible. score of $52.3 \pm 16.4$ on the Life-Space Assessment [10] provides evidence for mobility limitations within the study population in the month prior to admission.

None of the seven patients in the exercise group was able to participate in the in-hospital exercise program. Patients either were discharged before therapy could begin or were not available when the therapist visited because of other appointments. The average length of stay for study participants was 4.2 days, with 56 percent of patients (five of nine) being evaluated on the day of their discharge. While four of the seven patients participated in some component of the home exercise program, only one patient completed all 24 weeks of the program. For those who did not complete the exercise program, readmission to the hospital was a frequent barrier. Forty-three percent (three of seven) were admitted to the hospital at some time during the 24-week study period, and one participant died during the hospital stay.

After the study ended, because of our lack of success in recruiting and enrolling participants, we conducted phone interviews to elicit information about barriers to recruitment. We determined sample size by using theoretical saturation, where data collection and analysis occur simultaneously and enrollment ends when new interviews do not add new perspectives. Based on previous studies of this kind, we expected that small numbers of participants would be required [15]. A total of nine patients were interviewed by phone, three previous participants and six nonparticipants in the feasibility study. Table 4 presents the results of these interviews. The majority of patients interviewed (seven of nine) felt beginning to exercise either in the hospital or shortly thereafter was a good idea. While walking and strengthening exercises were thought to be the best form of exercise by the patients, two reported difficulty walking because of a lack of space and safe places to walk.

Table 2.

Medical conditions resulting in exclusion of potential subjects $(n=125)$.

\begin{tabular}{lc}
\multicolumn{1}{c}{ Condition } & Number of Patients (\%) \\
\hline Dementia (including Alzheimer’s disease) & $27(22)$ \\
Excessive Sedation and/or Confusion & $23(18)$ \\
Bed or Wheelchair Bound & $15(12)$ \\
Alcohol or Drug Addiction & $11(9)$ \\
Cancer with Life Expectancy $<1$ yr & $10(8)$ \\
Schizophrenia & $10(8)$ \\
Cardiac Dysfunction Resulting in Exercise Contraindications & $7(6)$ \\
Other Conditions Resulting in Inability to Safely Exercise & $22(18)$ \\
\hline \hline
\end{tabular}


JRRD, Volume 43, Number 7, 2006

Table 3.

Baseline characteristics and functional measures of study participants $(n=9)$.

\begin{tabular}{lc}
\hline \multicolumn{1}{c}{ Characteristic } & Mean \pm Standard Deviation or $\boldsymbol{N}(\%)$ \\
\hline Age (yr) & $70.2 \pm 3.1$ \\
Male & $9(100)$ \\
White & $7(78)$ \\
Married & $4(44)$ \\
Mini-Mental State Examination & $25.7 \pm 0.7$ \\
Timed Up and Go (s) & $18.9 \pm 6.1$ \\
Life-Space Assessment & $52.3 \pm 16.4$ \\
Geriatric Depression Scale (15-item) & $5.1 \pm 4.3$ \\
Acute Physiology Score at Randomization & $3.4 \pm 1.9$ \\
Charlson Comorbidity Index & $4.3 \pm 2.3$ \\
Number of Medications at Discharge & $9.4 \pm 4.7$ \\
\hline \hline
\end{tabular}

Table 4.

Participants' qualitative responses regarding exercise $(n=9)$.

\begin{tabular}{lc}
\hline \multicolumn{1}{c}{ Qualitative Response } & Number of Patients \\
\hline Reasons for Participation in Exercise Program & 2 \\
Feeling weak and want to get stronger & 1 \\
Exercise is good for you & 1 \\
Want to lose weight & 2 \\
Reasons to Not Participate in Exercise Program & 1 \\
Feeling unwell & 1 \\
Exercise makes me feel worse & \\
Doing enough exercise with activities of daily living & 7 \\
When to Begin Exercises & 2 \\
In hospital or shortly thereafter & 7 \\
When I feel better & 1 \\
Best Type of Exercise & 3 \\
Walking and strengthening & \\
Group exercise & \\
Having someone come to house to help with exercise & \\
\hline \hline
\end{tabular}

\section{DISCUSSION}

Our study determined the feasibility of an inpatient and in-home exercise program for older adults recovering from acute medical conditions in a Department of Veterans Affairs (VA) hospital. We encountered multiple barriers in our attempt to conduct this study. The first major barrier was the difficulty recruiting patients. Of those initially screened, $<2$ percent of the patients agreed to participate. Siebens et al. conducted an RCT with an in-hospital exercise intervention and a 1-month posthospitalization home exercise program and reported a similar success rate [16]. Of over 15,000 patients initially screened, only 300 were judged qualified and consented to participate in the study [16]. Barriers common to both studies include early hospital discharge, unacceptable medical diagnoses, and patient refusal.
Theoretically, an inpatient exercise program to prevent functional decline that often occurs with hospitalization is logical. However, these programs are difficult to implement. In the report by Siebens et al., which was conducted in a community-based hospital, 31 percent of the patients in the intervention group either received no exercise during hospitalization or $<50$ percent of the scheduled exercise sessions [16]. In our VA-based study, we were unable to implement an in-hospital exercise program. None of the 10 patients who consented was hospitalized long enough to begin an exercise program, and some were not available because of diagnostic tests and appointments with physicians or other healthcare professionals.

Other VA-based studies have had more success with recruitment. Oslin et al. were able to recruit 2,637 hospitalized older patients from medical and surgical units in nine VA medical centers for a study of the effects of care 
management for behavioral problems in older patients. They reported that 50 percent of those who were eligible and approached for screening consented to screening. Of those who consented, 63 percent met all inclusion criteria [17]. However, recruitment for exercise interventions, especially posthospitalization, has been more difficult. A study of low-intensity exercise and fall reduction noted that only 1 participant remained eligible after the physician screening of the 101 participants who were posthospitalization. Study participants were eliminated for medical conditions that made it unsafe to exercise, required human assistance to walk, or required hospital readmission within 2 months for evaluation or treatment [18]. This study demonstrates the difficulty encountered when recruiting from a population of acutely ill older adults.

One explanation for difficulty with recruitment of participants is staff inexperience. However, our inpatient recruiter had extensive experience with recruiting and coordinating a rigorous exercise intervention for older community-dwelling adults that required weight training three times a week for 6 months. This recruiter was highly effective in recruiting and achieved outstanding adherence with the exercise program. While we cannot exclude experience as a possible explanation, we do not believe it was the primary barrier in this study.

An alternative to an in-hospital exercise program is a simple walking program. One study, based in two community hospitals and one academic medical center, randomized patients to usual care versus an early ambulation program in a group of patients hospitalized with community-acquired pneumonia. The early ambulation group was discharged from the hospital 1.2 days sooner, on average, than those in the usual care group and had no increase in adverse effects or rehospitalizations [19]. The feasibility of a walking program was also demonstrated in a small pilot study done in a communitybased hospital, which used specially trained transporters to walk ward patients during slow periods, which included nights and weekends. On average, participants spent 2.4 days in the program, with an average of 5.6 walks per patient. Unfortunately, the study was too small to demonstrate the effects of the program on length of stay and functional decline [20]. This type of early walking intervention could be piloted within the VA hospital setting with potentially better success than the inpatient exercise program we have described.

A posthospitalization exercise intervention by Siebens et al. appeared to have a greater probability of success. Of the seven patients randomized to the rehabilitation group, 57 percent (four of seven) demonstrated some participation in the outpatient home exercise program. In the study, 42 percent of the subjects participated to some extent in the home exercise program. Two studies that included physical therapy for older adults posthospitalization reported positive results in their ability to perform instrumental ADL and walk [21-22]. Another study that used a progressive resistance muscle strengthening program also reported improvements in function and ambulation [23]. The patients who were interviewed expressed the belief that exercise, particularly walking and strengthening, would be beneficial especially if it was started just prior to hospital discharge or shortly after being discharged to home.

\section{CONCLUSIONS}

This study demonstrated the difficulties encountered with recruitment of medical participants during hospitalization and suggests an in-hospital exercise program may not be feasible within a VA acute-care setting. We were surprised by this finding, although similar challenges have been demonstrated in one study in a non-VA setting. While patients expressed interest, they frequently felt too ill or did not believe they were capable of exercising during the hospital stay. An in-home exercise program, perhaps implemented 2 to 4 weeks after hospital discharge with follow-up from trained staff, may have a higher likelihood of success and would be a reasonable target for additional research.

\section{ACKNOWLEDGMENTS}

We are indebted to the patients at the BVAMC who participated in this study.

This material was based on work supported in part by the VA, grant E2808-G. Dr. Brown is a recipient of a VA Research Career Development Award, grant E4-3842VA.

The authors have declared that no competing interests exist.

\section{REFERENCES}

1. Lamont CT, Sampson S, Matthias R, Kane R. The outcome of hospitalization for acute illness in the elderly. J Am Geriatr Soc. 1983;31(5):282-88. [PMID: 6841857] 
2. Hirsch CH, Sommers L, Olsen A, Mullen L, Winograd CH. The natural history of functional morbidity in hospitalized older patients. J Am Geriatr Soc. 1990;38(12):1296-1303. [PMID: 2123911]

3. Winograd $\mathrm{CH}$, Lindenberger EC, Chavez CM, Mauricio MP, Shi H, Bloch DA. Identifying hospitalized older patients at varying risk for physical performance decline: A new approach. J Am Geriatr Soc. 1997;45(5):604-9. [PMID: 9158583]

4. McVey LJ, Becker PM, Saltz CC, Feussner JR, Cohen HJ. Effect of a geriatric consultation team on functional status of elderly hospitalized patients. A randomized, controlled clinical trial. Ann Intern Med. 1989;110(1):79-84. [PMID: 2642284]

5. Warshaw GA, Moore JT, Friedman SW, Currie CT, Kennie DC, Kane WJ, Mears PA. Functional disability in the hospitalized elderly. JAMA. 1982;248(7):847-50.

[PMID: 6212699]

6. Covinsky KE, Palmer RM, Fortinsky RH, Counsell SR, Stewart AL, Kresevic D, Burant CJ, Landefeld CS. Loss of independence in activities of daily living in older adults hospitalized with medical illnesses: Increased vulnerability with age. J Am Geriatr Soc. 2003;51(4):451-58. [PMID: 12657063]

7. Sager MA, Franke T, Inouye SK, Landefeld CS, Morgan TM, Rudberg MA, Sebens H, Winograd CH. Functional outcomes of acute medical illness and hospitalization in older persons. Arch Intern Med. 1996;156(6):645-52. [PMID: 8629876]

8. Folstein MF, Folstein SE, McHugh PR. "Mini-mental state." A practical method for grading the cognitive status of patients for the clinician. J Psychiatr Res. 1975;12(3): 189-98. [PMID: 1202204]

9. Podsiadlo D, Richardson S. The timed "Up \& Go": A test of basic functional mobility for frail elderly persons. J Am Geriatr Soc. 1991;39(2):143-48. [PMID: 1991946]

10. Peel C, Sawyer Baker P, Roth DL, Brown CJ, Brodner EV, Allman RM. Assessing mobility in older adults: The UAB Study of Aging Life-Space Assessment. Phys Ther. 2005; 85(10):1008-19. [PMID: 16180950]

11. Yesavage JA, Brink TL, Rose TL, Lum O, Huang V, Adey $\mathrm{M}$, Leirer VO. Development and validation of a geriatric depression screening scale: A preliminary report. J Psychiatr Res. 1983;17(1):37-49. [PMID: 7183759]

12. Bohannon RW. A descriptive meta-analysis. J Geriatr Phys Ther. 2006;29(2):64-68. [PMID: 16914068]
13. Knaus WA, Draper EA, Wagner DP, Zimmerman JE. APACHE II: A severity of disease classification system. Crit Care Med. 1985;13(10):818-29. [PMID: 3928249]

14. Charlson ME, Pompei P, Ales KL, MacKenzie CR. A new method of classifying prognostic comorbidity in longitudinal studies: Development and validation. J Chronic Dis. 1986;40(5):373-83. [PMID: 3558716]

15. Tuckett AG. Qualitative research sampling: The very real complexities. Nurse Res. 2004;12(1):47-61. [PMID: 15493214]

16. Siebens H, Aronow H, Edwards D, Ghasemi Z. A randomized controlled trial of exercise to improve outcomes of acute hospitalization in older adults. J Am Geriatr Soc. 2000;48(12):1545-52. [PMID: 11129741]

17. Oslin DW, Thompson R, Kallan MJ, TenHave T, Blow FL, Bastani R, Gould RL, Maxwell AE, Rosansky J, Van Stone W, Jarvik L. Treatment effects from UPBEAT: A randomized trial of care management for behavioral health problems in hospitalized elderly patients. J Geriatr Psychiatry Neurol. 2004;17(2):99-106. [PMID: 15157351]

18. Morgan RO, Virnig BA, Duque M, Abdel-Moty E, Devito VA. Low-intensity exercise and reduction of the risk for falls among at-risk elders. J Gerontol A Biol Sci Med Sci. 2004;59(10):1062-67. [PMID: 15528779]

19. Mundy LM, Leet TL, Darst K, Schnitzler MA, Dunagan WC. Early mobilization of patients hospitalized with community-acquired pneumonia. Chest. 2003;124(3):883-89. [PMID: 12970012]

20. Tucker D, Molsberger SC, Clark A. Walking for wellness: A collaborative program to maintain mobility in hospitalized older adults. Geriatr Nurs. 2004;25(4):242-45. [PMID: 15311202]

21. Melin AL, Byren LO. Efficacy of the rehabilitation of elderly primary health care patients after short-stay hospital treatment. Med Care. 1992;30(11):1004-15. [PMID: 1331632]

22. Nikolaus T, Specht-Leible N, Bach M, Oster P, Schlierf G. A randomized trial of comprehensive geriatric assessment and home intervention in the care of hospitalized patients. Age Ageing. 1999;28(6):543-50. [PMID: 10604506]

23. Sullivan DH, Wall PT, Bariola JR, Bopp MM, Frost YM. Progressive resistance muscle strength training of hospitalized frail elderly. Am J Phys Med Rehabil. 2001;80(7): 503-9. [PMID: 11421518]

Submitted for publication April 11, 2006. Accepted in revised form September 26, 2006. 